\title{
Strong Convergence Theorem by a Hybrid Extragradient-like Approximation Method for Variational Inequalities and Fixed Point Problems
}

\author{
Lu-Chuan Ceng ${ }^{1}$, Nicolas Hadjisavvas ${ }^{2}$ and Ngai-Ching Wong ${ }^{3}$
}

\begin{abstract}
The purpose of this paper is to investigate the problem of finding a common element of the set of fixed points $F(S)$ of a nonexpansive mapping $S$ and the set of solutions $\Omega_{A}$ of the variational inequality for a monotone, Lipschitz continuous mapping $A$. We introduce a hybrid extragradient-like approximation method which is based on the well-known extragradient method and a hybrid (or outer approximation) method. The method produces three sequences which are shown to converge strongly to the same common element of $F(S) \cap \Omega_{A}$. As applications, the method provides an algorithm for finding the common fixed point of a nonexpansive mapping and a pseudocontractive mapping, or a common zero of a monotone Lipschitz continuous mapping and a maximal monotone mapping.
\end{abstract}

Keywords: Hybrid extragradient-like approximation method; variational inequality; fixed point; monotone mapping; nonexpansive mapping; demiclosedness principle.

Mathematics Subject Classification 2000: 47J20; 47H09.

\footnotetext{
${ }^{1}$ Department of Mathematics, Shanghai Normal University, Shanghai 200234, China. Email: zenglc@hotmail.com

${ }^{2}$ Corresponding author. Department of Product and Systems Design Engineering, University of the Aegean, 84100 Hermoupolis, Syros, Greece. Email: nhad@aegean.gr. The author was supported by grant no. 227- $\varepsilon$ of the Greek General Secretariat of Research and Technology.

${ }^{3}$ Department of Applied Mathematics, National Sun Yat-Sen University, Kaohsiung 804, Taiwan. Email: wong@math.nsysu.edu.tw
} 


\section{Introduction}

Let $H$ be a real Hilbert space with inner product $\langle\cdot, \cdot\rangle$ and norm $\|\cdot\|$, respectively. Let $C$ be a nonempty closed convex subset of $H$ and $A$ be a mapping of $C$ into $H$. Then $A$ is called monotone if

$$
\langle A x-A y, x-y\rangle \geq 0, \quad \forall x, y \in C .
$$

$A$ is called $\alpha$-inverse-strongly monotone (see $[4,12]$ ) if there exists a positive constant $\alpha$ such that

$$
\langle A x-A y, x-y\rangle \geq \alpha\|A x-A y\|^{2}, \quad \forall x, y \in C .
$$

$A$ is called $k$-Lipschitz continuous if there exists a positive constant $k$ such that

$$
\|A x-A y\| \leq k\|x-y\|, \quad \forall x, y \in C .
$$

It is clear that if $A$ is $\alpha$-inverse-strongly monotone, then $A$ is monotone and Lipschitz continuous.

In this paper, we consider the following variational inequality (for short, $\operatorname{VI}(A, C))$ : find $u \in C$ such that

$$
\langle A u, v-u\rangle \geq 0, \quad \forall v \in C .
$$

The set of solutions of the $\operatorname{VI}(C, A)$ is denoted by $\Omega_{A}$. A mapping $S: C \rightarrow C$ is called nonexpansive [7] if

$$
\|S x-S y\| \leq\|x-y\|, \quad \forall x, y \in C .
$$

We denote by $F(S)$ the set of fixed points of $S$, i.e., $F(S)=\{u \in C: S u=u\}$. A more restrictive class of maps are the contractive maps, i.e. maps $S: C \rightarrow C$ such that for some $\alpha \in(0,1)$,

$$
\|S x-S y\| \leq \alpha\|x-y\|, \quad \forall x, y \in C .
$$

Due to the many applications of the variational inequality problem to several branches of mathematics, but also to mechanics, economics etc, finding its solutions is a very important field of research. In some cases, as for strictly monotone operators $A$, the solution, if it exists, is unique. More generally the set of solutions $\Omega_{A}$ of a continuous monotone mapping $A$ is a convex subset of $C$. In such cases one is often interested in finding a solution that has some desirable properties. For instance, Antipin has investigated methods for finding a solution of a variational inequality that satisfies some additional inequality constraints $[1,2]$, in a finite-dimensional space. Takahashi and Toyoda [18] considered the problem of finding a solution of the variational inequality which is also a fixed point of some mapping, in an infinite-dimensional setting. More precisely, given a nonempty, closed and convex set $C \subseteq H$, a nonexpansive mapping $S: C \rightarrow C$ 
and an $\alpha$-inverse-strongly-monotone mapping $A: C \rightarrow H$, they introduced the following iterative scheme in order to find an element of $F(S) \cap \Omega_{A}$ :

$$
\left\{\begin{array}{l}
x_{0}=x \in C \\
x_{n+1}=\alpha_{n} x_{n}+\left(1-\alpha_{n}\right) S P_{C}\left(x_{n}-\lambda_{n} A x_{n}\right)
\end{array}\right.
$$

for all $n \geq 0$, where $\left\{\alpha_{n}\right\}$ is a sequence in $(0,1),\left\{\lambda_{n}\right\}$ is a sequence in $(0,2 \alpha)$, and $P_{C}$ is the metric projection of $H$ onto $C$. It is shown in [18] that if $F(S) \cap$ $\Omega_{A} \neq \emptyset$, then the sequence $\left\{x_{n}\right\}$ generated by (1) converges weakly to some $z \in F(S) \cap \Omega_{A}$. Later on, in order to achieve strong convergence to an element of $F(S) \cap \Omega_{A}$ under the same assumptions, Iiduka and Takahashi [10] modified the iterative scheme by using a hybrid method as follows:

$$
\left\{\begin{array}{l}
x_{0}=x \in C \\
y_{n}=\alpha_{n} x_{n}+\left(1-\alpha_{n}\right) S P_{C}\left(x_{n}-\lambda_{n} A x_{n}\right), \\
C_{n}=\left\{z \in C:\left\|y_{n}-z\right\| \leq\left\|x_{n}-z\right\|\right\} \\
Q_{n}=\left\{z \in C:\left\langle x_{n}-z, x-x_{n}\right\rangle \geq 0\right\} \\
x_{n+1}=P_{C_{n} \cap Q_{n}} x
\end{array}\right.
$$

for all $n \geq 0$, where $0 \leq \alpha_{n} \leq c<1$ and $0<a \leq \lambda_{n} \leq b<2 \alpha$. It is shown in [10] that if $F(S) \cap \Omega_{A} \neq \emptyset$, then the sequence $\left\{x_{n}\right\}$ converges strongly to $P_{F(S) \cap \Omega_{A}} x$.

The restriction of the above methods to the class of of $\alpha$-inverse strongly monotone mappings (i.e., mappings whose inverse is strongly monotone) excludes some important classes of mappings, as pointed out by Nadezhkina and Takahashi [14]. The so-called extragradient method, introduced in 1976 by Korpelevich [11] for a finite-dimensional space, provides an iterative process converging to a solution of $\operatorname{VI}(A, C)$ by only assuming that $C \subseteq \mathbb{R}^{n}$ is closed and convex and $A: C \rightarrow \mathbb{R}^{n}$ is monotone and $k$-Lipschitz continuous. The extragradient method was further extended to infinite dimensional spaces by many authors; see for instance the recent contributions of He, Yang and Yuan [8], Solodov and Svaiter [17], Ceng and Yao [6, 19] etc.

By modifying the extragradient method, Nadezhkina and Takahashi were able to show the following weak convergence result, for mappings $A$ that are only supposed to be monotone and $k$-Lipschitz:

Theorem 1 [13, Theorem 3.1] Let $C$ be a nonempty closed convex subset of a real Hilbert space $H$. Let $A: C \rightarrow H$ be a monotone and $k$-Lipschitz continuous mapping and $S: C \rightarrow C$ be a nonexpansive mapping such that $F(S) \cap \Omega_{A} \neq \emptyset$. Let $\left\{x_{n}\right\},\left\{y_{n}\right\}$ be the sequences generated by

$$
\left\{\begin{array}{l}
x_{0}=x \in C \\
y_{n}=P_{C}\left(x_{n}-\lambda_{n} A x_{n}\right), \\
x_{n+1}=\alpha_{n} x_{n}+\left(1-\alpha_{n}\right) S P_{C}\left(x_{n}-\lambda A y_{n}\right)
\end{array}\right.
$$

for all $n \geq 0$, where $\left\{\lambda_{n}\right\} \subset[a, b]$ for some $a, b \in(0,1 / k)$ and $\left\{\alpha_{n}\right\} \subset[c, d]$ for some $c, d \in(0,1)$. Then the sequences $\left\{x_{n}\right\},\left\{y_{n}\right\}$ converge weakly to the same point $z \in F(S) \cap \Omega_{A}$ where $z=\lim _{n \rightarrow \infty} P_{F(S) \cap \Omega_{A}} x_{n}$. 
Further, inspired by Nadezhkina and Takahashi's extragradient method [13], Ceng and Yao [5] also introduced and considered an extragradient-like approximation method which is based on the above extragradient method and the viscosity approximation method, and proved the following strong convergence result.

Theorem 2 [5, Theorem 3.1] Let $C$ be a nonempty closed convex subset of a real Hilbert space $H$. Let $f: C \rightarrow C$ be a contractive mapping with a contractive constant $\alpha \in(0,1), A: C \rightarrow H$ be a monotone, $k$-Lipschitz continuous mapping and $S: C \rightarrow C$ be a nonexpansive mapping such that $F(S) \cap \Omega_{A} \neq \emptyset$. Let $\left\{x_{n}\right\},\left\{y_{n}\right\}$ be the sequences generated by

$$
\left\{\begin{array}{l}
x_{0}=x \in C \\
y_{n}=\left(1-\gamma_{n}\right) x_{n}+\gamma_{n} P_{C}\left(x_{n}-\lambda_{n} A x_{n}\right) \\
x_{n+1}=\left(1-\alpha_{n}-\beta_{n}\right) x_{n}+\alpha_{n} f\left(y_{n}\right)+\beta_{n} S P_{C}\left(x_{n}-\lambda A y_{n}\right),
\end{array}\right.
$$

for all $n \geq 0$, where $\left\{\lambda_{n}\right\}$ is a sequence in $(0,1)$ with $\sum_{n=0}^{\infty} \lambda_{n}<\infty$, and $\left\{\alpha_{n}\right\},\left\{\beta_{n}\right\},\left\{\gamma_{n}\right\}$ are three sequences in $[0,1]$ satisfying the conditions:

(i) $\alpha_{n}+\beta_{n} \leq 1$ for all $n \geq 0$;

(ii) $\lim _{n \rightarrow \infty} \alpha_{n}=0, \quad \sum_{n=0}^{\infty} \alpha_{n}=\infty$;

(iii) $0<\liminf _{n \rightarrow \infty} \beta_{n} \leq \limsup _{n \rightarrow \infty} \beta_{n}<1$.

Then the sequences $\left\{x_{n}\right\},\left\{y_{n}\right\}$ converge strongly to the same point $q=P_{F(S) \cap \Omega_{A}} f(q)$ if and only if $\left\{A x_{n}\right\}$ is bounded and $\liminf _{n \rightarrow \infty}\left\langle A x_{n}, y-x_{n}\right\rangle \geq 0$ for all $y \in C$.

Very recently, by combining a hybrid-type method with an extragradienttype method, Nadezhkina and Takahashi [14] introduced the following iterative method for finding an element of $F(S) \cap \Omega_{A}$ and established the following strong convergence theorem.

Theorem 3 [14, Theorem 3.1] Let $C$ be a nonempty closed convex subset of a real Hilbert space $H$. Let $A: C \rightarrow H$ be a monotone and $k$-Lipschitz continuous mapping and let $S: C \rightarrow C$ be a nonexpansive mapping such that $F(S) \cap \Omega_{A} \neq \emptyset$. Let $\left\{x_{n}\right\},\left\{y_{n}\right\},\left\{z_{n}\right\}$ be sequences generated by

$$
\left\{\begin{array}{l}
x_{0}=x \in C, \\
y_{n}=P_{C}\left(x_{n}-\lambda_{n} A x_{n}\right), \\
z_{n}=\alpha_{n} x_{n}+\left(1-\alpha_{n}\right) S P_{C}\left(x_{n}-\lambda_{n} A y_{n}\right), \\
C_{n}=\left\{z \in C:\left\|z_{n}-z\right\| \leq\left\|x_{n}-z\right\|\right\}, \\
Q_{n}=\left\{z \in C:\left\langle x_{n}-z, x-x_{n}\right\rangle \geq 0\right\}, \\
x_{n+1}=P_{C_{n} \cap Q_{n}} x,
\end{array}\right.
$$

for all $n \geq 0$, where $\left\{\lambda_{n}\right\} \subset[a, b]$ for some $a, b \in(0,1 / k)$ and $\left\{\alpha_{n}\right\} \subset[0, c]$ for some $c \in[0,1)$. Then the sequences $\left\{x_{n}\right\},\left\{y_{n}\right\},\left\{z_{n}\right\}$ converge strongly to the same point $q=P_{F(S) \cap \Omega_{A}} x$.

In this paper, we introduce a hybrid extragradient-like approximation method which is based on the above extragradient method and hybrid (or outer approx- 
imation) method, i.e.,

$$
\left\{\begin{array}{l}
x_{0} \in C, \\
y_{n}=\left(1-\gamma_{n}\right) x_{n}+\gamma_{n} P_{C}\left(x_{n}-\lambda_{n} A x_{n}\right) \\
z_{n}=\left(1-\alpha_{n}-\beta_{n}\right) x_{n}+\alpha_{n} y_{n}+\beta_{n} S P_{C}\left(x_{n}-\lambda_{n} A y_{n}\right), \\
C_{n}=\left\{z \in C:\left\|z_{n}-z\right\|^{2} \leq\left\|x_{n}-z\right\|^{2}+\left(3-3 \gamma_{n}+\alpha_{n}\right) b^{2}\left\|A x_{n}\right\|^{2}\right\} \\
Q_{n}=\left\{z \in C:\left\langle x_{n}-z, x_{0}-x_{n}\right\rangle \geq 0\right\} \\
x_{n+1}=P_{C_{n} \cap Q_{n}} x_{0}
\end{array}\right.
$$

for all $n \geq 0$, where $\left\{\lambda_{n}\right\}$ is a sequence in $[a, b]$ with $a>0$ and $b<\frac{1}{2 k}$, and $\left\{\alpha_{n}\right\},\left\{\beta_{n}\right\},\left\{\gamma_{n}\right\}$ are three sequences in $[0,1]$ satisfying the conditions:

(i) $\alpha_{n}+\beta_{n} \leq 1$ for all $n \geq 0$;

(ii) $\lim _{n \rightarrow \infty} \alpha_{n}=0$;

(iii) $\liminf _{n \rightarrow \infty} \beta_{n}>0$

(iv) $\lim _{n} \gamma_{n}=1$ and $\gamma_{n}>3 / 4$ for all $n \geq 0$.

It is shown that the sequences $\left\{x_{n}\right\},\left\{y_{n}\right\},\left\{z_{n}\right\}$ generated by the above hybrid extragradient-like approximation method are well-defined and converge strongly to the same point $q=P_{F(S) \cap \Omega_{A}} x$. Using this theorem, we construct an iterative process for finding a common fixed point of two mappings, one of which is nonexpansive and the other taken from the more general class of Lipschitz pseudocontractive mappings.

In the next section we will recall some basic notions and results. In Section 3 we present and prove our main theorem. The last section is devoted to some applications.

\section{Preliminaries}

Given a nonempty, closed and convex subset $C$ of a Hilbert space $H$, for any $x \in H$ there exists a unique element $P_{C} x \in C$ which is nearest to $x$, i.e. for all $y \in C$,

$$
\left\|x-P_{C} x\right\| \leq\|x-y\|
$$

The projection operator $P_{C}: H \rightarrow C$ is nonexpansive on $H$ : For every $x, y \in H$,

$$
\left\|P_{C} x-P_{C} y\right\| \leq\|x-y\| .
$$

In addition, it has the following properties: For every $x \in H$ and $y \in C$,

$$
\|x-y\|^{2} \geq\left\|x-P_{C} x\right\|^{2}+\left\|y-P_{C} x\right\|^{2} ;
$$

also,

$$
\left\langle x-P_{C} x, y-P_{C} x\right\rangle \leq 0 .
$$

Assume that $A$ is monotone and continuous. Then the solutions of the variational inequality $\mathrm{VI}(A, C)$ can be characterized as solutions of the so-called Minty variational inequality:

$$
x^{*} \in \Omega_{A} \Leftrightarrow\left\langle A x, x-x^{*}\right\rangle \geq 0, \quad \forall x \in C .
$$


We will also make use of Browder's demiclosedness principle, cf. for instance [16]. Let us denote by $I$ the identity operator in $H$.

Proposition 4 Let $C \subseteq H$ be closed and convex. Assume that $S: C \rightarrow H$ is nonexpansive. If $S$ has a fixed point, then $I-S$ is demiclosed; that is, whenever $\left\{x_{n}\right\}$ is a sequence in $C$ converging weakly to some $x \in C$ and the sequence $\left\{(I-S) x_{n}\right\}$ converges strongly to some $y \in H$, it follows that $(I-S) x=y$.

A mapping $T: C \rightarrow C$ is called pseudocontractive if and only if for all $x, y \in C$,

$$
\langle T x-T y, x-y\rangle \leq\|x-y\|^{2} .
$$

It is clear that any contractive mapping is pseudocontractive. Also, it is easy to see that $T$ is pseudocontractive if and only if the mapping $A=I-T$ is monotone [3].

A multivalued mapping $B: H \rightarrow 2^{H}$ is called monotone if for all $x, y \in H$, $x^{*} \in T x$ and $y^{*} \in T y$ one has $\left\langle y^{*}-x^{*}, y-x\right\rangle \geq 0$. Such a mapping is called maximal monotone if it has no proper monotone extension, i.e., if $B_{1}: H \rightarrow 2^{H}$

is monotone and $B x \subseteq B_{1} x$ for all $x \in H$, then $B=B_{1}$. If $B$ is maximal monotone, then for each $r>0$ and $x \in H$ there exists a unique element $z \in H$ such that $(I+r B) z=x$. This element is denoted by $J_{r}^{B} x$. The mapping $J_{r}^{B}$ thus defined is called the resolvent of $B[9]$.

\section{The main convergence result}

In this section we define an iterative process and prove its convergence to a member of $F(S) \cap \Omega_{A}$, where $S$ is nonexpansive and $A$ is monotone and $k$ Lipschitz continuous.

Theorem 5 Let $C$ be a nonempty closed convex subset of a real Hilbert space $H$, $A: C \rightarrow H$ be a monotone, $k$-Lipschitz continuous mapping and let $S: C \rightarrow C$ be a nonexpansive mapping such that $F(S) \cap \Omega_{A} \neq \emptyset$. We define inductively the sequences $\left\{x_{n}\right\},\left\{y_{n}\right\},\left\{z_{n}\right\}$ by

$$
\begin{aligned}
x_{0} & \in C, \\
y_{n} & =\left(1-\gamma_{n}\right) x_{n}+\gamma_{n} P_{C}\left(x_{n}-\lambda_{n} A x_{n}\right), \\
z_{n} & =\left(1-\alpha_{n}-\beta_{n}\right) x_{n}+\alpha_{n} y_{n}+\beta_{n} S P_{C}\left(x_{n}-\lambda_{n} A y_{n}\right), \\
C_{n} & =\left\{z \in C:\left\|z_{n}-z\right\|^{2} \leq\left\|x_{n}-z\right\|^{2}+\left(3-3 \gamma_{n}+\alpha_{n}\right) b^{2}\left\|A x_{n}\right\|^{2}\right\}, \\
Q_{n} & =\left\{z \in C:\left\langle x_{n}-z, x_{0}-x_{n}\right\rangle \geq 0\right\}, \\
x_{n+1} & =P_{C_{n} \cap Q_{n}} x_{0}
\end{aligned}
$$

for all $n \geq 0$, where $\left\{\lambda_{n}\right\}$ is a sequence in $[a, b]$ with $a>0$ and $b<\frac{1}{2 k}$, and $\left\{\alpha_{n}\right\},\left\{\beta_{n}\right\},\left\{\gamma_{n}\right\}$ are three sequences in $[0,1]$ satisfying the conditions:

(i) $\alpha_{n}+\beta_{n} \leq 1$ for all $n \geq 0$;

(ii) $\lim _{n \rightarrow \infty} \alpha_{n}=0$; 
(iii) $\liminf _{n \rightarrow \infty} \beta_{n}>0$

(iv) $\lim _{n} \gamma_{n}=1$ and $\gamma_{n}>3 / 4$ for all $n \geq 0$.

Then the sequences $\left\{x_{n}\right\},\left\{y_{n}\right\},\left\{z_{n}\right\}$ are well-defined and converge strongly to the same point $q=P_{F(S) \cap \Omega_{A}} x_{0}$.

Proof. We divide the proof into several steps.

Step 1. Assuming that $x_{n}$ is a well-defined element of $C$ for some $n \in \mathbb{N}$, we show that $F(S) \cap \Omega_{A} \subset C_{n}$.

Since $x_{n}$ is defined, $y_{n}, z_{n}$ are obviously well-defined elements of $C$. Let $x^{*} \in F(S) \cap \Omega_{A}$ be arbitrary. Set $t_{n}=P_{C}\left(x_{n}-\lambda_{n} A y_{n}\right)$ for all $n \geq 0$. Taking $x=x_{n}-\lambda_{n} A y_{n}$ and $y=x^{*}$ in (5), we obtain

$$
\begin{aligned}
\left\|t_{n}-x^{*}\right\|^{2} & \leq\left\|x_{n}-\lambda_{n} A y_{n}-x^{*}\right\|^{2}-\left\|x_{n}-\lambda_{n} A y_{n}-t_{n}\right\|^{2} \\
& =\left\|x_{n}-x^{*}\right\|^{2}+2 \lambda_{n}\left\langle A y_{n}, x^{*}-y_{n}\right\rangle+2 \lambda_{n}\left\langle A y_{n}, y_{n}-t_{n}\right\rangle-\left\|x_{n}-t_{n}\right\|^{2} .
\end{aligned}
$$

Since by (7) we have $\left\langle A y_{n}, y_{n}-x^{*}\right\rangle \geq 0$, we deduce

$$
\begin{aligned}
\left\|t_{n}-x^{*}\right\|^{2} & \leq\left\|x_{n}-x^{*}\right\|^{2}-\left\|x_{n}-t_{n}\right\|^{2}+2 \lambda_{n}\left\langle A y_{n}, y_{n}-t_{n}\right\rangle \\
& =\left\|x_{n}-x^{*}\right\|^{2}-\left\|\left(x_{n}-y_{n}\right)+\left(y_{n}-t_{n}\right)\right\|^{2}+2 \lambda_{n}\left\langle A y_{n}, y_{n}-t_{n}\right\rangle \\
& =\left\|x_{n}-x^{*}\right\|^{2}-\left\|x_{n}-y_{n}\right\|^{2}-\left\|y_{n}-t_{n}\right\|^{2}+2\left\langle x_{n}-\lambda_{n} A y_{n}-y_{n}, t_{n}-y_{n}\right\rangle .
\end{aligned}
$$

We estimate the last term, using $y_{n}=\left(1-\gamma_{n}\right) x_{n}+\gamma_{n} P_{C}\left(x_{n}-\lambda_{n} A x_{n}\right)$ :

$$
\begin{aligned}
& \left\langle x_{n}-\lambda_{n} A y_{n}-y_{n}, t_{n}-y_{n}\right\rangle \\
& =\left\langle x_{n}-\lambda_{n} A x_{n}-y_{n}, t_{n}-y_{n}\right\rangle+\lambda_{n}\left\langle A x_{n}-A y_{n}, t_{n}-y_{n}\right\rangle \\
& \leq\left\langle x_{n}-\lambda_{n} A x_{n}-\left(1-\gamma_{n}\right) x_{n}-\gamma_{n} P_{C}\left(x_{n}-\lambda_{n} A x_{n}\right), t_{n}-y_{n}\right\rangle+\lambda_{n}\left\|A x_{n}-A y_{n}\right\|\left\|t_{n}-y_{n}\right\| \\
& \leq \gamma_{n}\left\langle x_{n}-\lambda_{n} A x_{n}-P_{C}\left(x_{n}-\lambda_{n} A x_{n}\right), t_{n}-y_{n}\right\rangle \\
& \quad-\left(1-\gamma_{n}\right) \lambda_{n}\left\langle A x_{n}, t_{n}-y_{n}\right\rangle+\lambda_{n} k\left\|x_{n}-y_{n}\right\|\left\|t_{n}-y_{n}\right\| .
\end{aligned}
$$

In addition, using properties (6) and (4) of the projection $P_{C}\left(x_{n}-\lambda_{n} A x_{n}\right)$ we obtain

$$
\begin{aligned}
& \left\langle x_{n}-\lambda_{n} A x_{n}-P_{C}\left(x_{n}-\lambda_{n} A x_{n}\right), t_{n}-y_{n}\right\rangle \\
& =\left\langle x_{n}-\lambda_{n} A x_{n}-P_{C}\left(x_{n}-\lambda_{n} A x_{n}\right), t_{n}-\left(1-\gamma_{n}\right) x_{n}-\gamma_{n} P_{C}\left(x_{n}-\lambda_{n} A x_{n}\right\rangle\right. \\
& =\left(1-\gamma_{n}\right)\left\langle x_{n}-\lambda_{n} A x_{n}-P_{C}\left(x_{n}-\lambda_{n} A x_{n}\right), t_{n}-x_{n}\right\rangle \\
& \quad+\gamma_{n}\left\langle x_{n}-\lambda_{n} A x_{n}-P_{C}\left(x_{n}-\lambda_{n} A x_{n}\right), t_{n}-P_{C}\left(x_{n}-\lambda_{n} A x_{n}\right)\right\rangle \\
& \leq\left(1-\gamma_{n}\right)\left\|x_{n}-\lambda_{n} A x_{n}-P_{C}\left(x_{n}-\lambda_{n} A x_{n}\right)\right\|\left\|t_{n}-x_{n}\right\| \\
& \leq\left(1-\gamma_{n}\right) \lambda_{n}\left\|A x_{n}\right\|\left(\left\|t_{n}-y_{n}\right\|+\left\|y_{n}-x_{n}\right\|\right) .
\end{aligned}
$$


Gathering (8), (9), (10) and using $\gamma_{n} \leq 1$ and $\lambda_{n} \leq b$ we find

$$
\begin{aligned}
\left\|t_{n}-x^{*}\right\|^{2} \leq & \left\|x_{n}-x^{*}\right\|^{2}-\left\|x_{n}-y_{n}\right\|^{2}-\left\|y_{n}-t_{n}\right\|^{2} \\
& +2 \gamma_{n}\left(1-\gamma_{n}\right) b\left\|A x_{n}\right\|\left(\left\|t_{n}-y_{n}\right\|+\left\|y_{n}-x_{n}\right\|\right) \\
& +2\left(1-\gamma_{n}\right) b\left\|A x_{n}\right\|\left\|t_{n}-y_{n}\right\|+2 b k\left\|x_{n}-y_{n}\right\|\left\|t_{n}-y_{n}\right\| \\
\leq & \left\|x_{n}-x^{*}\right\|^{2}-\left\|x_{n}-y_{n}\right\|^{2}-\left\|y_{n}-t_{n}\right\|^{2} \\
& +\left(1-\gamma_{n}\right)\left(2 b^{2}\left\|A x_{n}\right\|^{2}+\left\|t_{n}-y_{n}\right\|^{2}+\left\|y_{n}-x_{n}\right\|^{2}\right) \\
& +\left(1-\gamma_{n}\right)\left(b^{2}\left\|A x_{n}\right\|^{2}+\left\|t_{n}-y_{n}\right\|^{2}\right)+b k\left(\left\|x_{n}-y_{n}\right\|^{2}+\left\|t_{n}-y_{n}\right\|^{2}\right) \\
= & \left\|x_{n}-x^{*}\right\|^{2}-\left\|x_{n}-y_{n}\right\|^{2}\left(\gamma_{n}-b k\right) \\
& -\left\|y_{n}-t_{n}\right\|^{2}\left(2 \gamma_{n}-1-b k\right)+3\left(1-\gamma_{n}\right) b^{2}\left\|A x_{n}\right\|^{2} .
\end{aligned}
$$

Using our assumptions $b<\frac{1}{2 k}$ and $\gamma_{n}>3 / 4$, we obtain that for all $n \in \mathbb{N}$,

$$
\left\|t_{n}-x^{*}\right\|^{2} \leq\left\|x_{n}-x^{*}\right\|^{2}+3\left(1-\gamma_{n}\right) b^{2}\left\|A x_{n}\right\|^{2} .
$$

Also, using again (7) and properties of $P_{C}$, we obtain

$$
\begin{aligned}
\left\|y_{n}-x^{*}\right\|^{2} & =\left\|\left(1-\gamma_{n}\right)\left(x_{n}-x^{*}\right)+\gamma_{n}\left(P_{C}\left(x_{n}-\lambda_{n} A x_{n}\right)-x^{*}\right)\right\|^{2} \\
& \leq\left(1-\gamma_{n}\right)\left\|x_{n}-x^{*}\right\|^{2}+\gamma_{n}\left\|P_{C}\left(x_{n}-\lambda_{n} A x_{n}\right)-P_{C} x^{*}\right\|^{2} \\
& \leq\left(1-\gamma_{n}\right)\left\|x_{n}-x^{*}\right\|^{2}+\gamma_{n}\left\|x_{n}-x^{*}-\lambda_{n} A x_{n}\right\|^{2} \\
& =\left(1-\gamma_{n}\right)\left\|x_{n}-x^{*}\right\|^{2}+\gamma_{n}\left[\left\|x_{n}-x^{*}\right\|^{2}-2 \lambda_{n}\left\langle A x_{n}, x_{n}-x^{*}\right\rangle+\lambda_{n}^{2}\left\|A x_{n}\right\|^{2}\right] \\
& \leq\left\|x_{n}-x^{*}\right\|^{2}+b^{2}\left\|A x_{n}\right\|^{2}
\end{aligned}
$$

Since $S$ is nonexpansive and $x^{*} \in F(S)$ we have $\left\|S t_{n}-x^{*}\right\| \leq\left\|t_{n}-x^{*}\right\|$. Thus, (13) and (12) imply that

$$
\begin{aligned}
\left\|z_{n}-x^{*}\right\|^{2}= & \left\|\left(1-\alpha_{n}-\beta_{n}\right) x_{n}+\alpha_{n} y_{n}+\beta_{n} S t_{n}-x^{*}\right\|^{2} \\
\leq & \left(1-\alpha_{n}-\beta_{n}\right)\left\|x_{n}-x^{*}\right\|^{2}+\alpha_{n}\left\|y_{n}-x^{*}\right\|^{2}+\beta_{n}\left\|S t_{n}-x^{*}\right\|^{2} \\
\leq & \left(1-\alpha_{n}-\beta_{n}\right)\left\|x_{n}-x^{*}\right\|^{2}+\alpha_{n}\left[\left\|x_{n}-x^{*}\right\|^{2}+b^{2}\left\|A x_{n}\right\|^{2}\right] \\
& +\beta_{n}\left[\left\|x_{n}-x^{*}\right\|^{2}+3\left(1-\gamma_{n}\right) b^{2}\left\|A x_{n}\right\|^{2}\right] \\
= & \left\|x_{n}-x^{*}\right\|^{2}+\left(3-3 \gamma_{n}+\alpha_{n}\right) b^{2}\left\|A x_{n}\right\|^{2} .
\end{aligned}
$$

Consequently, $x^{*} \in C_{n}$. Hence $F(S) \cap \Omega_{A} \subset C_{n}$.

Step 2. We show that the sequence $\left\{x_{n}\right\}$ is well-defined and $F(S) \cap \Omega_{A} \subset$ $C_{n} \cap Q_{n}$ for all $n \geq 0$.

We show this assertion by mathematical induction. For $n=0$ we have $Q_{0}=C$. Hence by Step 1 we obtain $F(S) \cap \Omega_{A} \subset C_{0} \cap Q_{0}$. Assume that $x_{k}$ is defined and $F(S) \cap \Omega_{A} \subset C_{k} \cap Q_{k}$ for some $k \geq 0$. Then $y_{k}, z_{k}$ are well-defined elements of $C$. Note that $C_{k}$ is a closed convex subset of $C$ since

$$
C_{k}=\left\{z \in C:\left\|z_{k}-x_{k}\right\|^{2}+2\left\langle z_{k}-x_{k}, x_{k}-z\right\rangle \leq\left(3-3 \gamma_{k}+\alpha_{k}\right) b^{2}\left\|A x_{k}\right\|^{2}\right\} .
$$


Also, it is obvious that $Q_{k}$ is closed and convex. Thus, $C_{k} \cap Q_{k}$ is a closed convex subset, which is nonempty since by assumption it contains $F(S) \cap \Omega_{A}$. Consequently, $x_{k+1}=P_{C_{k} \cap Q_{k}} x_{0}$ is well-defined.

The definition of $x_{k+1}$ and of $Q_{k+1}$ imply that $C_{k} \cap Q_{k} \subseteq Q_{k+1}$. Hence, $F(S) \cap \Omega_{A} \subseteq Q_{k+1}$. Using Step 1 we infer that $F(S) \cap \Omega_{A} \subseteq C_{k+1} \cap Q_{k+1}$.

Step 3. We show that the following statements hold:

(1) $\left\{x_{n}\right\}$ is bounded, $\lim _{n \rightarrow \infty}\left\|x_{n}-x_{0}\right\|$ exists, and $\lim _{n \rightarrow \infty}\left(x_{n+1}-x_{n}\right)=0$;

(2) $\lim _{n \rightarrow \infty}\left(z_{n}-x_{n}\right)=0$.

Indeed, take any $x^{*} \in F(S) \cap \Omega_{A}$. Using $x_{n+1}=P_{C_{n} \cap Q_{n}} x_{0}$ and $x^{*} \in$ $F(S) \cap \Omega_{A} \subset C_{n} \cap Q_{n}$, we obtain

$$
\left\|x_{n+1}-x_{0}\right\| \leq\left\|x^{*}-x_{0}\right\|, \quad \forall n \geq 0 .
$$

Therefore, $\left\{x_{n}\right\}$ is bounded and so is $\left\{A x_{n}\right\}$ due to the Lipschitz continuity of $A$. From the definition of $Q_{n}$ it is clear that $x_{n}=P_{Q_{n}} x_{0}$. Since $x_{n+1} \in$ $C_{n} \cap Q_{n} \subset Q_{n}$, we have

$$
\left\|x_{n+1}-x_{n}\right\|^{2} \leq\left\|x_{n+1}-x_{0}\right\|^{2}-\left\|x_{n}-x_{0}\right\|^{2} \quad \forall n \geq 0 .
$$

In particular, $\left\|x_{n+1}-x_{0}\right\| \geq\left\|x_{n}-x_{0}\right\|$ hence $\lim _{n \rightarrow \infty}\left\|x_{n}-x_{0}\right\|$ exists. Then (17) implies that

$$
\lim _{n \rightarrow \infty}\left(x_{n+1}-x_{n}\right)=0 .
$$

Since $x_{n+1} \in C_{n}$, we have

$$
\left\|z_{n}-x_{n+1}\right\|^{2} \leq\left\|x_{n}-x_{n+1}\right\|^{2}+\left(3-3 \gamma_{n}+\alpha_{n}\right) b^{2}\left\|A x_{n}\right\|^{2} .
$$

Since $\left\{A x_{n}\right\}$ is bounded and $\lim _{n \rightarrow \infty} \gamma_{n}=1, \lim _{n \rightarrow \infty} \alpha_{n}=0$, we deduce that $\lim _{n \rightarrow \infty}\left(z_{n}-x_{n+1}\right)=0$. Combining with (18) we infer that $\lim _{n \rightarrow \infty}\left(z_{n}-\right.$ $\left.x_{n}\right)=0$.

Step 4. We show that the following statements hold:

(1) $\lim _{n \rightarrow \infty}\left(x_{n}-y_{n}\right)=0$;

(2) $\lim _{n \rightarrow \infty}\left(S x_{n}-x_{n}\right)=0$.

Indeed, from inequalities (14) and (15) we infer

$$
\begin{aligned}
\left\|z_{n}-x^{*}\right\|^{2}-\left\|x_{n}-x^{*}\right\|^{2} & \leq\left(-\alpha_{n}-\beta_{n}\right)\left\|x_{n}-x^{*}\right\|^{2}+\alpha_{n}\left\|y_{n}-x^{*}\right\|^{2}+\beta_{n}\left\|S t_{n}-x^{*}\right\|^{2} \\
& \leq\left(3-3 \gamma_{n}+\alpha_{n}\right) b^{2}\left\|A x_{n}\right\|^{2} .
\end{aligned}
$$

Since $\alpha_{n} \rightarrow 0, \gamma_{n} \rightarrow 1$, and $\left\{x_{n}\right\},\left\{A x_{n}\right\},\left\{y_{n}\right\}$ are bounded, we deduce from (19) that

$$
\lim _{n \rightarrow+\infty} \beta_{n}\left(\left\|S t_{n}-x^{*}\right\|^{2}-\left\|x_{n}-x^{*}\right\|^{2}\right)=0 .
$$


Using $\liminf \operatorname{in}_{n \rightarrow+\infty} \beta_{n}>0$ we get $\lim _{n \rightarrow+\infty}\left(\left\|S t_{n}-x^{*}\right\|^{2}-\left\|x_{n}-x^{*}\right\|^{2}\right)=0$. Then (12) implies

$$
\begin{aligned}
\lim _{n \rightarrow+\infty}\left(\left\|S t_{n}-x^{*}\right\|^{2}-\left\|x_{n}-x^{*}\right\|^{2}\right) & \leq \lim _{n \rightarrow+\infty}\left(\left\|t_{n}-x^{*}\right\|^{2}-\left\|x_{n}-x^{*}\right\|^{2}\right) \\
& \leq \lim _{n \rightarrow+\infty} 3\left(1-\gamma_{n}\right) b^{2}\left\|A x_{n}\right\|^{2}=0
\end{aligned}
$$

thus, $\lim _{n \rightarrow+\infty}\left(\left\|t_{n}-x^{*}\right\|^{2}-\left\|x_{n}-x^{*}\right\|^{2}\right)=0$. Now we rewrite (11) as

$\left\|x_{n}-y_{n}\right\|^{2}\left(\gamma_{n}-b k\right)+\left\|y_{n}-t_{n}\right\|^{2}\left(2 \gamma_{n}-1-b k\right) \leq\left\|x_{n}-x^{*}\right\|^{2}-\left\|t_{n}-x^{*}\right\|^{2}+3\left(1-\gamma_{n}\right) b^{2}\left\|A x_{n}\right\|^{2}$.

We deduce that

$$
\lim _{n \rightarrow+\infty}\left[\left\|x_{n}-y_{n}\right\|^{2}\left(\gamma_{n}-b k\right)+\left\|y_{n}-t_{n}\right\|^{2}\left(2 \gamma_{n}-1-b k\right)\right]=0 .
$$

Our assumptions on $\lambda_{n}$ and $\gamma_{n}$ imply that $\gamma_{n}-b k>1 / 4$ and $2 \gamma_{n}-1-b k>$ $\frac{1}{2}-b k>0$. Consequently, $\lim _{n \rightarrow+\infty}\left(x_{n}-y_{n}\right)=\lim _{n \rightarrow+\infty}\left(y_{n}-t_{n}\right)=0$. Hence, $\lim _{n \rightarrow+\infty}\left(x_{n}-t_{n}\right)=0$. Using that $S$ is nonexpansive, we get $\lim _{n \rightarrow+\infty}\left(S x_{n}-\right.$ $\left.S t_{n}\right)=0$.

We rewrite the definition of $z_{n}$ as

$$
z_{n}-x_{n}=-\alpha_{n} x_{n}+\alpha_{n} y_{n}+\beta_{n}\left(S t_{n}-x_{n}\right) .
$$

From $\lim _{n \rightarrow+\infty}\left(z_{n}-x_{n}\right)=0, \lim _{n \rightarrow+\infty} \alpha_{n}=0$, the boundedness of $x_{n}, y_{n}$ and $\liminf \inf _{n \rightarrow+\infty} \beta_{n}>0$ we infer that $\lim _{n \rightarrow+\infty}\left(S t_{n}-x_{n}\right)=0$. Thus finally $\lim _{n \rightarrow+\infty}\left(S x_{n}-x_{n}\right)=0$.

Step 5. We claim that $\omega_{w}\left(x_{n}\right) \subset F(S) \cap \Omega_{A}$, where $\omega_{w}\left(x_{n}\right)$ denotes the weak $\omega$-limit set of $\left\{x_{n}\right\}$, i.e.,

$\omega_{w}\left(x_{n}\right)=\left\{u \in H:\left\{x_{n_{j}}\right\}\right.$ converges weakly to $u$ for some subsequence $\left\{n_{j}\right\}$ of $\left.\{n\}\right\}$.

Indeed, since $\left\{x_{n}\right\}$ is bounded, it has a subsequence which converges weakly to some point in $C$ and hence $\omega_{w}\left(x_{n}\right) \neq \emptyset$. Let $u \in \omega_{w}\left(x_{n}\right)$ be arbitrary. Then there exists a subsequence $\left\{x_{n_{j}}\right\} \subset\left\{x_{n}\right\}$ which converges weakly to $u$. Since we also have $\lim _{j \rightarrow \infty}\left(x_{n_{j}}-S x_{n_{j}}\right)=0$, from the demiclosedness principle it follows that $(I-S) u=0$. Thus $u \in F(S)$. We now show that $u \in \Omega_{A}$.

Since $t_{n}=P_{C}\left(x_{n}-\lambda_{n} A y_{n}\right)$, for every $x \in C$ we have

$$
\left\langle x_{n}-\lambda_{n} A y_{n}-t_{n}, t_{n}-x\right\rangle \geq 0
$$

hence

$$
\left\langle x-t_{n}, A y_{n}\right\rangle \geq\left\langle x-t_{n}, \frac{x_{n}-t_{n}}{\lambda_{n}}\right\rangle .
$$

Combining with monotonicity of $A$ we obtain

$$
\begin{aligned}
\left\langle x-t_{n}, A x\right\rangle & \geq\left\langle x-t_{n}, A t_{n}\right\rangle \\
& =\left\langle x-t_{n}, A t_{n}-A y_{n}\right\rangle+\left\langle x-t_{n}, A y_{n}\right\rangle \\
& \geq\left\langle x-t_{n}, A t_{n}-A y_{n}\right\rangle+\left\langle x-t_{n}, \frac{x_{n}-t_{n}}{\lambda_{n}}\right\rangle .
\end{aligned}
$$


Since $\lim _{n \rightarrow+\infty}\left(x_{n}-t_{n}\right)=\lim _{n \rightarrow+\infty}\left(y_{n}-t_{n}\right)=0, A$ is Lipschitz continuous and $\lambda_{n}>a>0$ we deduce that

$$
\langle x-u, A x\rangle=\lim _{n_{j} \rightarrow+\infty}\left\langle x-t_{n_{j}}, A x\right\rangle \geq 0, \quad \forall x \in C .
$$

Then (7) entails that $u \in F(S) \cap \Omega_{A}$.

Step 6. We show that $\left\{x_{n}\right\},\left\{y_{n}\right\},\left\{z_{n}\right\}$ converge strongly to the same point $q=P_{F(S) \cap \Omega_{A}} x_{0}$.

Assume that $\left\{x_{n}\right\}$ does not converge strongly to $q$. Then there exists $\varepsilon>0$ and a subsequence $\left\{x_{n_{j}}\right\} \subset\left\{x_{n}\right\}$ such that $\left\|x_{n_{j}}-q\right\|>\varepsilon$ for all $j$. Without loss of generality we may assume that $\left\{x_{n_{j}}\right\}$ converges weakly to some point $u$. By Step 5, $u \in F(S) \cap \Omega_{A}$. Using $q=P_{F(S) \cap \Omega_{A}} x_{0}$, the weak lower semicontinuity of $\|\cdot\|$, and relation (16) for $x^{*}=q$, we obtain

$$
\left\|q-x_{0}\right\| \leq\left\|u-x_{0}\right\| \leq \liminf _{j \rightarrow \infty}\left\|x_{n_{j}}-x_{0}\right\|=\lim _{n \rightarrow+\infty}\left\|x_{n}-x_{0}\right\| \leq\left\|q-x_{0}\right\| .
$$

It follows that $\left\|q-x_{0}\right\|=\left\|u-x_{0}\right\|$, hence $u=q$ since $q$ is the unique element in $F(S) \cap \Omega_{A}$ that minimizes the distance from $x_{0}$. Also, (20) implies $\lim _{j \rightarrow \infty}\left\|x_{n_{j}}-x_{0}\right\|=\left\|q-x_{0}\right\|$. Since $\left\{x_{n_{j}}-x_{0}\right\}$ converges weakly to $q-x_{0}$, this shows that $\left\{x_{n_{j}}-x_{0}\right\}$ converges strongly to $q-x_{0}$, and hence $\left\{x_{n_{j}}\right\}$ converges strongly to $q$, a contradiction.

Thus, $\left\{x_{n}\right\}$ converges strongly to $q$. It is easy to see that $\left\{y_{n}\right\},\left\{z_{n}\right\}$ converge strongly to the same point $q$.

\section{Applications}

If one takes $\alpha_{n}=0, \beta_{n}=1$ and $\gamma_{n}=1$ for all $n \in \mathbb{N}$ in Theorem 5 , then one finds the following simpler theorem:

Theorem 6 Let $C$ be a nonempty closed convex subset of a real Hilbert space $H$, $A: C \rightarrow H$ be a monotone, $k$-Lipschitz continuous mapping and let $S: C \rightarrow C$ be a nonexpansive mapping such that $F(S) \cap \Omega_{A} \neq \emptyset$. We define inductively the sequences $\left\{x_{n}\right\},\left\{y_{n}\right\},\left\{z_{n}\right\}$ by

$$
\begin{aligned}
x_{0} & \in C, \\
y_{n} & =P_{C}\left(x_{n}-\lambda_{n} A x_{n}\right), \\
z_{n} & =S P_{C}\left(x_{n}-\lambda_{n} A y_{n}\right), \\
C_{n} & =\left\{z \in C:\left\|z_{n}-z\right\|^{2} \leq\left\|x_{n}-z\right\|^{2}\right\}, \\
Q_{n} & =\left\{z \in C:\left\langle x_{n}-z, x_{0}-x_{n}\right\rangle \geq 0\right\}, \\
x_{n+1} & =P_{C_{n} \cap Q_{n}} x_{0}
\end{aligned}
$$

for all $n \geq 0$, where $\left\{\lambda_{n}\right\}$ is a sequence in $[a, b]$ with $a>0$ and $b<\frac{1}{2 k}$. Then the sequences $\left\{x_{n}\right\},\left\{y_{n}\right\},\left\{z_{n}\right\}$ are well-defined and converge strongly to the same point $q=P_{F(S) \cap \Omega_{A}} x_{0}$. 
However, relations like (15) suggest that, as is often the case, taking more general sequences $\left\{\alpha_{n}\right\},\left\{\beta_{n}\right\}$ and $\left\{\gamma_{n}\right\}$ might improve the rate of convergence to a solution.

Taking $S=I, \alpha_{n}=0$ and $\beta_{n}=1$ in Theorem 5, one finds the following theorem providing an algorithm to find the solution of a variational inequality:

Theorem 7 Let $C$ be a nonempty closed convex subset of a real Hilbert space $H$, $A: C \rightarrow H$ be a monotone, $k$-Lipschitz continuous mapping such that $\Omega_{A} \neq \emptyset$. We define inductively the sequences $\left\{x_{n}\right\},\left\{y_{n}\right\},\left\{z_{n}\right\}$ by

$$
\begin{aligned}
x_{0} & \in C, \\
y_{n} & =\left(1-\gamma_{n}\right) x_{n}+\gamma_{n} P_{C}\left(x_{n}-\lambda_{n} A x_{n}\right), \\
z_{n} & =P_{C}\left(x_{n}-\lambda_{n} A y_{n}\right), \\
C_{n} & =\left\{z \in C:\left\|z_{n}-z\right\|^{2} \leq\left\|x_{n}-z\right\|^{2}+\left(3-3 \gamma_{n}\right) b^{2}\left\|A x_{n}\right\|^{2}\right\}, \\
Q_{n} & =\left\{z \in C:\left\langle x_{n}-z, x_{0}-x_{n}\right\rangle \geq 0\right\}, \\
x_{n+1} & =P_{C_{n} \cap Q_{n}} x_{0}
\end{aligned}
$$

for all $n \geq 0$, where $\left\{\lambda_{n}\right\}$ is a sequence in $[a, b]$ with $a>0$ and $b<\frac{1}{2 k}$, and $\left\{\gamma_{n}\right\}$ is a sequence in $[0,1]$ such that $\lim _{n} \gamma_{n}=1$ and $\gamma_{n}>3 / 4$ for all $n \geq 0$.

Then the sequences $\left\{x_{n}\right\},\left\{y_{n}\right\},\left\{z_{n}\right\}$ are well-defined and converge strongly to the same point $q=P_{\Omega_{A}} x_{0}$.

Taking $\gamma_{n}=1$ and $\alpha_{n}=0$ in Theorem 5, one recovers the main result of [14]. If in addition one puts $A=0$, one recovers the main result of [15] on an algorithm to find the fixed point of a nonexpansive mapping.

Another consequence of Theorem 5 is the following.

Theorem 8 Let $H$ be a real Hilbert space, $A: H \rightarrow H$ be monotone and $k$ Lipschitz, and $S: H \rightarrow H$ be nonexpansive, such that $F(S) \cap A^{-1}\{0\} \neq \emptyset$. Define the sequences $\left\{x_{n}\right\},\left\{y_{n}\right\}$ and $\left\{z_{n}\right\}$ by

$$
\left\{\begin{array}{l}
x_{0} \in H \\
y_{n}=x_{n}-\lambda_{n} A x_{n} \\
z_{n}=\left(1-\beta_{n}\right) x_{n}-\alpha_{n} \lambda_{n} A x_{n}+\beta_{n} S\left(x_{n}-\frac{\lambda_{n}}{\gamma_{n}} A y_{n}\right) \\
C_{n}=\left\{z \in C:\left\|z_{n}-z\right\|^{2} \leq\left\|x_{n}-z\right\|^{2}+\left(3-3 \gamma_{n}+\alpha_{n}\right) b^{2}\left\|A x_{n}\right\|^{2}\right\} \\
Q_{n}=\left\{z \in C:\left\langle x_{n}-z, x_{0}-x_{n}\right\rangle \geq 0\right\} \\
x_{n+1}=P_{C_{n} \cap Q_{n}} x_{0}
\end{array}\right.
$$

for all $n \geq 0$, where $\left\{\lambda_{n}\right\}$ is a sequence in $[a, b]$ with $a>0$ and $b<\frac{1}{4 k}$, and $\left\{\alpha_{n}\right\},\left\{\beta_{n}\right\},\left\{\gamma_{n}\right\}$ are three sequences in $[0,1]$ satisfying the conditions:

(i) $\alpha_{n}+\beta_{n} \leq 1$ for all $n \geq 0$;

(ii) $\lim _{n \rightarrow \infty} \alpha_{n}=0$;

(iii) $\liminf _{n \rightarrow \infty} \beta_{n}>0$

(iv) $\lim _{n} \gamma_{n}=1$ and $\gamma_{n}>3 / 4$ for all $n \geq 0$.

Then the sequences $\left\{x_{n}\right\},\left\{y_{n}\right\}$ and $\left\{z_{n}\right\}$ are well-defined and converge strongly to the same point $q=P_{F(S) \cap A^{-1}\{0\}} x_{0}$. 
Proof. We set $\lambda_{n}^{\prime}=\lambda_{n} / \gamma_{n}$. Then $a \leq \lambda_{n}^{\prime}<\frac{4}{3} \lambda_{n}<2 b<\frac{1}{2} k$. Thus we can apply Theorem 5 for this sequence, and for $C=H$. We have $P_{H}=I$ and $\Omega_{A}=A^{-1}\{0\}$. Then Theorem 5 guarantees that the sequences $\left\{x_{n}\right\},\left\{y_{n}\right\}$ and and $\left\{z_{n}\right\}$ converge to $q=P_{F(S) \cap A^{-1}\{0\}} x_{0}$, where

$$
\begin{aligned}
y_{n} & =x_{n}-\gamma_{n} \lambda_{n}^{\prime} A x_{n}=x_{n}-\lambda_{n} A x_{n}, \\
z_{n} & =\left(1-\alpha_{n}-\beta_{n}\right) x_{n}+\alpha_{n} y_{n}+\beta_{n} S\left(x_{n}-\lambda_{n}^{\prime} A y_{n}\right) \\
& =\left(1-\beta_{n}\right) x_{n}-\alpha_{n} \lambda_{n} A x_{n}+\beta_{n} S\left(x_{n}-\frac{\lambda_{n}}{\gamma_{n}} A y_{n}\right) .
\end{aligned}
$$

Because of the relations that exist between monotone operators and nonexpansive mappings, Theorem 5 can also be applied for finding the common zeros of two monotone mappings, or the common fixed points of two mappings. For example, suppose that $A: H \rightarrow H$ is a monotone, Lipschitz continuous mapping and $B: H \rightarrow H$ is a maximal monotone mapping. Assume that the set of common zeros $A^{-1}(0) \cap B^{-1}(0)$ is nonempty. Theorem 5 can be applied to find an element of $A^{-1}(0) \cap B^{-1}(0)$ as follows. It is known that for any $r>0$ the resolvent $J_{r}^{B}$ of $B$ is nonexpansive [9]; also, if we set $C=H$ then $F\left(J_{r}^{B}\right)=B^{-1}(0)$ while $\Omega_{A}=A^{-1}(0)$. Thus, by applying Theorem 5 to the mappings $A$ and $J_{r}^{B}$ we can find an element of $\Omega_{A} \cap F\left(J_{r}^{B}\right)=A^{-1}(0) \cap B^{-1}(0)$.

Likewise, assume that $C \subseteq H$ is nonempty, closed convex, $T: C \rightarrow C$ is pseudocontractive and Lipschitz, and $S: C \rightarrow C$ is nonexpansive, such that $F(T) \cap F(S) \neq \emptyset$. We can find an element of $F(T) \cap F(S)$ as follows. If we set $A=I-T$ then it is known that $A$ is monotone and Lipschitz [3]. Also, it is easy to see that $\Omega_{A}=F(T)$. Indeed, if $u \in F(T)$ then $A u=0$ so that $u \in \Omega_{A}$. Conversely, if $u \in \Omega_{A}$ then

$$
\langle u-T u, y-u\rangle \geq 0, \quad \forall y \in C .
$$

Setting $y=T u$ we get $\langle u-T u, u-T u\rangle \leq 0$, i.e., $u \in F(T)$. Consequently, Theorem 5 can be applied to the mappings $A$ and $S$ to produce sequences converging to an element of $\Omega_{A} \cap F(S)=F(T) \cap F(S)$.

\section{References}

[1] A.S. Antipin, Methods for solving variational inequalities with related constraints, Comput. Math. Math. Phys., 40, 1239-1254 (2000).

[2] A.S. Antipin and F.P. Vasiliev, Regularized prediction method for solving variational inequalities with an inexactly given set, Comput. Math. Math. Phys., 44, 750-758 (2004).

[3] F.E. Browder, Existence of periodic solutions for nonlinear equations of evolution, Proc. Nat. Acad. Sc. USA, 55, 1100-1103 (1965).

[4] F.E. Browder and W.V. Petryshyn, Construction of fixed points of nonlinear mappings in Hilbert space, J. Math. Anal. Appl., 20, 197-228 (1967). 
[5] L.C. Ceng and J.C. Yao, An extragradient-like approximation method for variational inequality problems and fixed point problems, Appl. Math. Comput., 190, 205-215 (2007).

[6] L.C. Ceng and J.C. Yao, On the convergence analysis of inexact hybrid extragradient proximal point algorithms for maximal monotone operators, J. Comput. Appl. Math., 217, 326-338 (2007).

[7] K. Geobel and W.A. Kirk, Topics on Metric Fixed-Point Theory, Cambridge University Press, Cambridge, England (1990).

[8] B.-S. He, Z.-H. Yang and X.-M. Yuan, An approximate proximalextragradient type method for monotone variational inequalities, J. Math. Anal. Appl., 300 (2004) 362-374.

[9] S. Hu and N.S. Papageorgiou, Handbook of Multivalued Analysis, vol. I: Theory, Kluwer Academic Publishers, Dordrecht (1997).

[10] H. Iiduka and W. Takahashi, Strong convergence theorem by a hybrid method for nonlinear mappings of nonexpansive and monotone type and applications, Adv. Nonlinear Var. Inequal., 9, 1-10 (2006).

[11] G.M. Korpelevich, The extragradient method for finding saddle points and other problems, Matecon, 12, 747-756 (1976).

[12] F. Liu and M.Z. Nashed, Regularization of nonlinear ill-posed variational inequalities and convergence rates, Set-Valued Anal., 6, 313-344 (1998).

[13] N. Nadezhkina and W. Takahashi, Weak convergence theorem by an extragradient method for nonexpansive mappings and monotone mappings, J. Optim. Theory Appl., 128, 191-201 (2006).

[14] N. Nadezhkina and W. Takahashi, Strong convergence theorem by a hybrid method for nonexpansive mappings and Lipschitz-continuous monotone mappings, SIAM J. Optim., 16, 1230-1241 (2006).

[15] K. Nakajo and W. Takahashi, Strong convergence theorems for nonexpansive mappings and nonexpansive semigroups, J. Math. Anal. Appl., 279, $372-379$ (2003).

[16] Z. Opial, Weak convergence $f$ the sequence of successive approximations for nonlinear mappings, Bull. Amer. Math. Soc. 73, 591-597 (1967).

[17] M.V. Solodov and B.F. Svaiter, An inexact hybrid generalized proximal point algorithm and some new results on the theory of Bregman functions, Math. Oper. Res., 25, 214-230 (2000).

[18] W. Takahashi and M. Toyoda, Weak convergence theorems for nonexpansive mappings and monotone mappings, J. Optim. Theory Appl., 118, 417-428 (2003). 
[19] L.C. Zeng and J.C. Yao, Strong convergence theorem by an extragradient method for fixed point problems and variational inequality problems, Taiwan. J. Math., 10, 1293-1303 (2006). 